\title{
Methodology Report: The Impaired Theory of Mind in Autism Spectrum Disorders and the Possible Remediative Role of Transcranial Direct Current Stimulation
}

Fatemeh Shamsi ${ }^{1}$, Samira Hosseini ${ }^{1}$, Mahshid Tahamtan ${ }^{1}$, Masoumeh Bayat ${ }^{1 *}$

1. Department of Neuroscience, School of Advanced Medical Sciences and Technologies, Shiraz University of Medical Sciences, Shiraz, Iran.

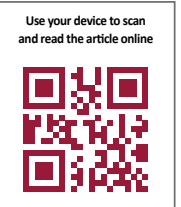

Citation: Shamsi F, Hosseini S, Tahamtan M, Bayat M. The Impaired Theory of Mind in Autism Spectrum Disorders and the Possible Remediative Role of Transcranial Direct Current Stimulation. Journal of Advanced Medical Sciences and Applied Technologies. 2017; 3(3):175-178. https://doi.org/10.32598/jamsat.3.3.175

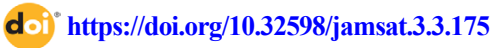

Article info:

Received: 26 Feb. 2017

Accepted: 31 May 2017

\section{Keywords:}

Autism spectrum disorder, Transcranial direct current stimulation, Theory of mind

\begin{abstract}
A BSTRACT
Autism Spectrum Disorders (ASDs) are complex neurodevelopmental conditions with the characteristics including impairments in social interaction and communication, and restricted patterns of behaviors or interests. Lack of Theory of Mind (TOM), which is considered as the core concept of social interaction, was suggested as the underlying deficit of social, behavioral, and communicative impairments of ASD. Transcranial Direct Current Stimulation (tDCS), as a non-invasive brain stimulation technique, indicated promising results for the treatment of psychiatric disorders. The present methodology report aimed at proposing a design to study the possible effects of tDCS on TOM and cognitive empathy in ASD.
\end{abstract}

\section{Introduction}

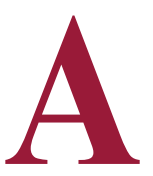

utism Spectrum Disorders (ASDs), which predominantly resulted from neurodevelopmental deficits, are complex heritable conditions associated with multiple genes and express variable phenotypes [1]. However, the exact etiopathophysiology of ASD still remained unknown. According to the Centers for Disease Control and Prevention (CDC), the prevalence of ASD was estimated as 1 in 68 in 2014 [2]. Impairments in social interaction, communication, and restricted patterns of behaviors or interest are regarded as classical characteristics of ASD [2]. Social interaction largely depends on the understanding of others' mental states; and social abilities such as Theory of Mind (TOM) and cognitive empathy are considered as its core concepts [3]. Lack of TOM was suggested as the underlying deficit of social, behavioral, and communicative impairments of ASD, whereby many studies on ASD were focused during the recent years [4].

The individuals' conscious awareness on their behaviors, guided by their thoughts and beliefs regardless of the accuracy of their beliefs, is defined as TOM [5]. As such, TOM enables people to conduct the interpersonal world in an effective manner and make reasonable explanations for others' behaviors [6]. 
Many researchers believe that the ability of understanding others' perspectives or mental states, known as cognitive empathy, is another concept closely linked to TOM [7-9]. In addition, Walter (2012) proposed the concept of cognitive empathy as an emotional component of TOM [10].

Studies, which attempted to define the underlying mechanisms of ASD, reported some structural and functional differences in the brain of subjects with ASD, compared with healthy individuals. Based on a recent investigation, some disruptions in the brain circuits of subjects with ASD were reported, including the hypoactivation and decreased functional connectivity in regions considered as parts of TOM network, compared with Typically Developing Controls (TDC) [6]. Furthermore, it was shown that the Temporoparietal Junction (TPJ), Temporal Poles (TP), and medial prefrontal cortex are also involved in TOM-related tasks [11]. There were some reports on the asymmetry between the 2 hemispheres in some brain structures related to language and social function in individuals with ASD, compared with transcranial Direct Current Stimulation (tDCS). For instance, larger volume in right hemisphere was noted in ASD subjects. There were also some reports on the hypoactivation of specific brain regions involved in face processing and social cognition in brain imaging studies on ASD cases [12]

In clinical studies, behavioral interventions were regarded among the most effective therapies for ASD with an efficacy rate up to $48 \%$. However, this type of therapy is time-consuming and costly; hence, not affordable for many affected subjects and families [13].

During the recent years, non-invasive transcranial brain stimulation techniques were used for the treatment

A Theory of Mind

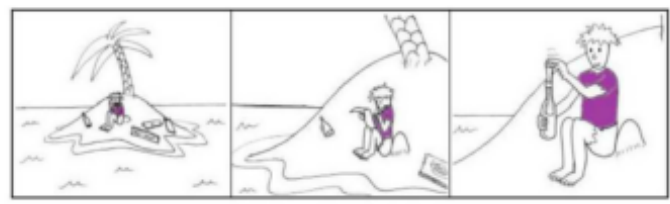

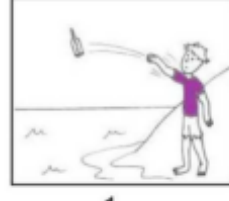

1

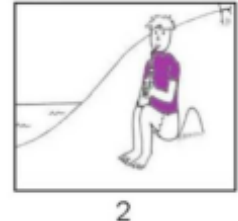

of psychiatric disorders yielding promising results. tDCS is among such techniques, which is considered as safe, easy, and comfortable; while providing effective focal modulation in target cortical brain areas [14].

In an elegant recent investigation, empathy abilities and TOM in normal subjects were found to be reduced following the cathodal tDCS-stimulation on the right TPJ (rTPJ) [3]. Quantitative Electroencephalography (QEEG) is a typically applied non-invasive tool to measure neural correlates at cortical level in various neurocognitive predicaments including ASD $[15,16]$. The present methodology report aimed at proposing a design to study the possible effects of tDCS on TOM and cognitive empathy in ASD cases.

\section{Materials and Methods}

\section{Participants}

A proper sample size of right-handed male children with high-functioning ASD, aged 10 to 12 years and 15 normally developing children matched by age, gender, and IQ, as controls, would be recruited. Children can be recruited voluntarily among the members of the Autism Association of Shiraz who had been diagnosed with autism by a neurologist; normal subjects are also recruited from healthy volunteers. The experimental subjects might be randomly assigned to the real anodal and the control "sham" groups.

\section{Experimental procedure}

QEEG signals of participants should be recorded, while performing the TOM and cognitive empathy tasks derived from Mai et al. (2016), on computer before and after intervention with a 16- channel electroencepha-

\section{Empathy}

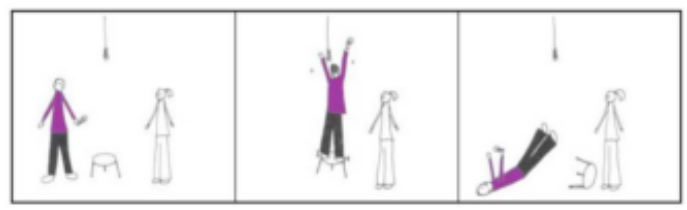

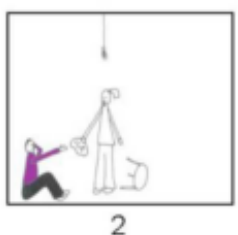

JAMSAT

Figure 1. Examples of stimuli from 2 conditions: A. Theory of mind, B. Empathy; Reproduced from “Using tDCS to Explore the Role of the Right Temporo-Parietal Junction in Theory of Mind and Cognitive Empathy" [3] 


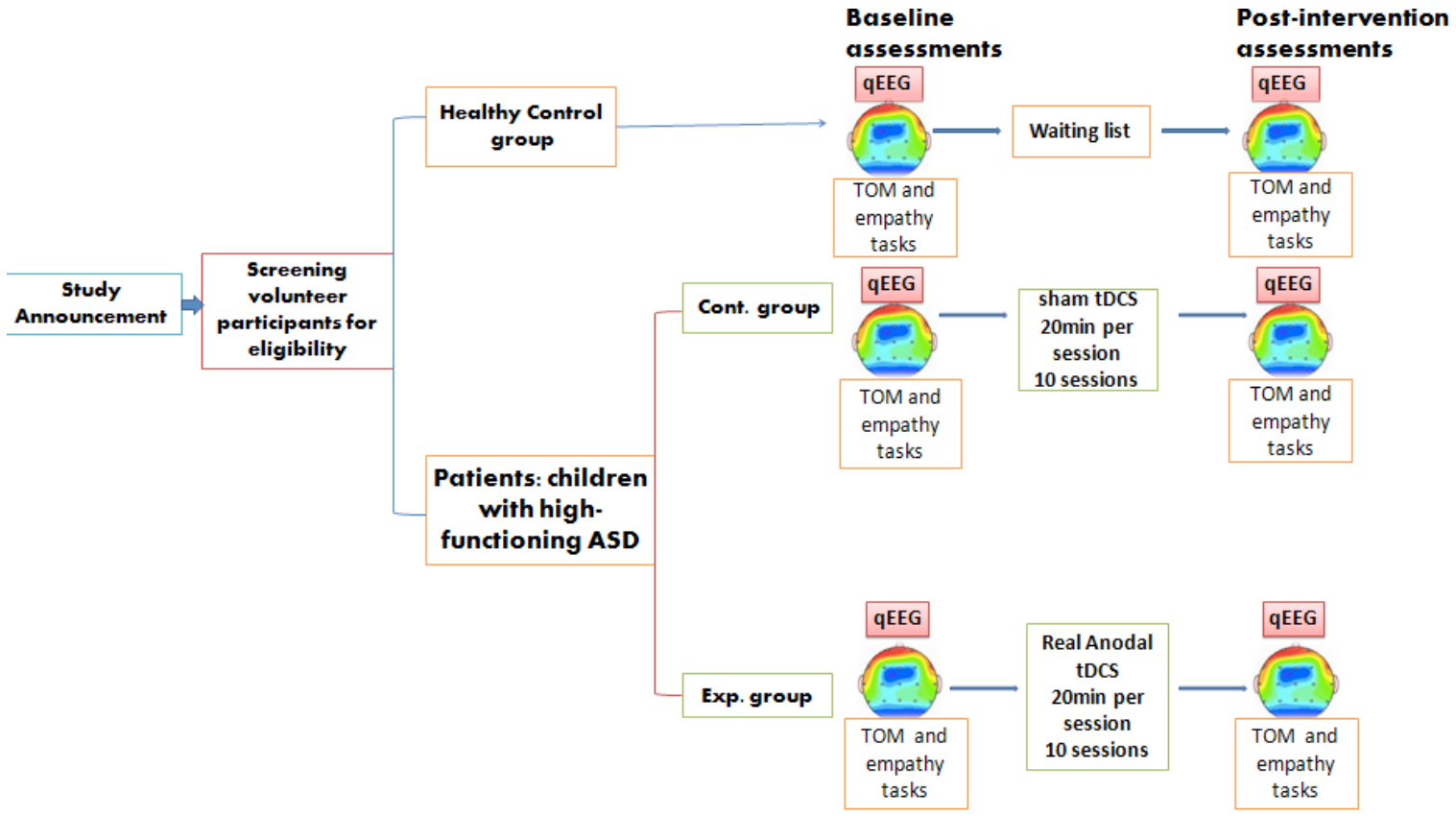

Figure 2. The diagram summaries the whole protocol from participants recruiting to final assessments.

ASD: Autism Spectrum Disorder; TOM: Theory of Mind; tDCS: transcranial Direct Current Stimulation; QEEG: Quantitative Electroencephalography

logram [3]. Connectivity map, local power-spectrum, would be compared between 3 groups for investigating QEEG pattern improvement in the experimental group.

Two conditions might be included in the task: TOM and cognitive empathy. Figure 1 shows examples of stimuli from each condition. It is suggested that in the TOM and cognitive empathy conditions, participants be asked to infer the character's intention or emotion, respectively. The stories of TOM describe 1 character only, while cognitive empathy describes 2 characters. It seems that 20 comic strips depicting a short story are enough for 4 blocks, each block containing 5 comic strips regarding the same condition. Accordingly, each condition is observed twice, while each strip is noted once. The sequence of blocks and comic strips in each block must be counterbalanced. Each block starts with an introductory question within 6 seconds indicating the type of inference (TOM condition: "What will the main character do next?"; Cognitive empathy condition: "What will make the main character feel better?"). Each cartoon is proposed to be shown for 6 seconds, in follows 2 further cartoons showing the possible outcome are imposed on the bottom of the screen for 4.5 seconds. Subjects make a choice between the 2 possible outcomes of the stories by pushing the button as soon as possible. Accuracy and RTs are recorded for each cartoon. A score of 1 refers to a correct answer, while a score of 0 assigns to be wrong [3].

The stimulation is induced through a saline-soaked pair of surface sponge electrodes ( $35 \mathrm{~cm}^{2}$ in size). Some of the previous studies showed the role of rTPJ in such cognitive capacities [3,17]; hence, the rTPJ might be the area of interest to study the effect of anodal tDCS on TOM and cognitive empathy in high-functioning children with ASD. To stimulate the rTPJ, the anodal electrode should be placed between $\mathrm{CP} 6$ and $\mathrm{C} 6$, according to the international 10-20 EEG system and previous functional Magnetic Resonance Imaging (fMRI) studies. The reference electrode can be located over the left cheek. A relatively weak current $(1.5 \mathrm{~mA})$ is suggested to be constantly delivered for 20 minutes each session. In the sham group, the electrode is placed over the rTPJ for 20 minutes, but the stimulation only lasts for 15 seconds [3]. A 10-session intervention, applied 5 days a week for 2 weeks, seems sufficient for both groups. Figure 2 illustrates the model of intervention protocol and assessments.

\section{Conclusion}

Regarding the promising effects of tDCS in neurological conditions including psychological disorders, it seems that this new technique can be considered as a probable effective intervention for improving communi- 
cative skills in ASD provided that the patterns and locations of electrophysiological deviations are detected as accurate as possible.

\section{Acknowledgments}

This research did not receive any specific grant from funding agencies in the public, commercial, or not-forprofit sectors.

\section{Conflict of Interest}

The authors have no financial or nonfinancial conflicts of interest.

\section{References}

[1] Johnson CP, Myers SM. Identification and evaluation of children with autism spectrum disorders. Paediatrics. 2007; 120(5):1183-215. doi: 10.1542/peds.2007-2361

[2] Coben R, Sherlin L, Hudspeth W, McKeon K, Ricca R. Connectivity-Guided EEG Biofeedback for Autism Spectrum Disorder: Evidence of Neurophysiological Changes. NeuroRegulation. 2014; 1(2):109-30. doi: 10.15540/nr.1.2.109

[3] Mai X, Zhang W, Hu X, Zhen Z, Xu Z, Zhang J, et al. Using tDCS to explore the role of the right temporo-parietal junction in theory of mind and cognitive empathy. Frontiers in Psychology. 2016; 7. doi: 10.3389/fpsyg.2016.00380

[4] Hutchins TL, Prelock PA, Chace W. Test-retest reliability of a theory of mind task battery for children with autism spectrum disorders. Focus on autism and other developmental disabilities. 2008; 23(4):195-206. doi: 10.1177/1088357608322998

[5] Peterson CC, Garnett M, Kelly A, Attwood T. Everyday social and conversation applications of theory-of-mind understanding by children with autism-spectrum disorders or typical development. European Child \& Adolescent Psychiatry. 2008; 18(2):105-15. doi: 10.1007/s00787-008-0711-y

[6] Kana RK, Maximo JO, Williams DL, Keller TA, Schipul SE, Cherkassky VL, et al. Aberrant functioning of the theory-ofmind network in children and adolescents with autism. Molecular Autism. 2015; 6(1). doi: 10.1186/s13229-015-0052-x

[7] Preston SD, de Waal FBM. Empathy: Its ultimate and proximate bases. Behavioral and Brain Sciences. 2001; 25(1):1-20. doi: $10.1017 / \mathrm{s} 0140525 \times 02000018$

[8] Shamay-Tsoory SG, Aharon-Peretz J, Perry D. Two systems for empathy: a double dissociation between emotional and cognitive empathy in inferior frontal gyrus versus ventromedial prefrontal lesions. Brain. 2009; 132(3):617-27. doi 10.1093/brain/awn279

[9] Schnell K, Bluschke S, Konradt B, Walter H. Functional relations of empathy and mentalizing: An fMRI study on the neural basis of cognitive empathy. NeuroImage. 2011; 54(2):174354. doi: 10.1016/j.neuroimage.2010.08.024
[10] Walter H. Social Cognitive Neuroscience of Empathy: Concepts, Circuits, and Genes. Emotion Review. 2012; 4(1):9-17. doi: $10.1177 / 1754073911421379$

[11] Kanske P, Böckler A, Singer T. Models, mechanisms and moderators dissociating empathy and theory of mind. Current Topics in Behavioral Neurosciences. 2015; 193-206. doi: 10.1007/7854_2015_412

[12] Amatachaya A, Auvichayapat N, Patjanasoontorn N Suphakunpinyo C, Ngernyam N, Aree-uea B, et al. Effect of anodal transcranial direct current stimulation on autism: a randomized double-blind crossover trial. Behavioural Neurology. 2014; 2014:1-7. doi: 10.1155/2014/173073

[13] Pineda JA, Friedrich EVC, LaMarca K. Neurorehabilitation of social dysfunctions: A model-based neurofeedback approach for low and high-functioning autism. Frontiers in Neuroengineering. 2014; 7:29. doi: 10.3389/fneng.2014.00029

[14] D’Urso G, Ferrucci R, Bruzzese D, Pascotto A, Priori A, Altamura CA, et al. Transcranial direct current stimulation for autistic disorder. Biological Psychiatry. 2014; 76(5):5-6. doi: 10.1016/j.biopsych.2013.11.009

[15] Coben R. The Importance of Electroencephalogram Assessment for Autistic Disorders. Biofeedback. Association for Applied Psychophysiology and Biofeedback; 2009; 37(2):71-80. doi: $10.5298 / 1081-5937-37.2 .71$

[16] Billeci L, Sicca F, Maharatna K, Apicella F, Narzisi A, Campatelli $G$, et al. On the application of quantitative EEG for characterizing autistic brain: a systematic review. Frontiers in $\mathrm{Hu}-$ man Neuroscience. 2013; 7. doi: 10.3389/fnhum.2013.00442

[17] Santiesteban I, Banissy MJ, Catmur C, Bird G. Enhancing social ability by stimulating right temporoparietal junction. Current Biology. 2012; 22(23):2274-7. doi: 10.1016/j. cub.2012.10.018 